\title{
Various Aspects of the Spectroscopy of PAHs (Polycyclic Aromatic Hydrocarbons)
}

\author{
Philippe Bréchignac \\ Laboratoire de Photophysique Moléculaire, CNRS, Batiment 210, \\ Université Paris-Sud, F-91405 ORSAY CEDEX (France)
}

\begin{abstract}
The exploitation of the recent observations (in particular by ISO) of the near infrared emission bands (UIBs) requires a good knowledge of the spectroscopic properties of the main proposed carriers, i.e. the Polycyclic Aromatic Hydrocarbons, which are expected to act as efficient $\mathrm{UV} /$ visible to infrared free-flying light converters.

The charge state of the interstellar PAHs strongly affects their spectroscopy. The laboratory study of the electronic transitions of PAH cations in the gas phase enables to address important questions, like their possible contribution to the Diffuse Interstellar Bands (visible and near-IR absorption), and the mechanism responsible for electronic to vibrational intramolecular energy conversion.

Microcanonical simulations based on hybrid quantum/classical theoretical methods reveal how strongly affected are the infrared intensities by both the charge state and the vibrational temperature.
\end{abstract}

\section{Introduction}

\subsection{Historical: from the UIBs to the PAHs}

The historically called "Unidentified Infrared emission Bands" (UIBs) have been recognized as associated to carbonaceous compounds as early as the late seventies (Knacke 1977). A major step was achieved by Sellgren (1984) by introducing the concept of transient heating of very small dust grains. The image of "free flying" PAHs which was proposed shortly after brought a consistent picture for explaining both the spectral coincidence of the band wavelengths with the vibrational frequencies of aromatic compounds and the excitation mechanism (Léger \& Puget 1984; Allamandola, Tielens \& Barker 1985). Absorption of individual UV photons by isolated PAHs, followed by intramolecular energy conversion between electronic states and (ultimately) the vibrational manifold of the $\mathrm{PAH}$ ground electronic state, was recognized as an efficient process able to account for most of the pre-ISO observations. It is, however, of extreme importance to realize that, according to this mechanism, the interstellar PAHs remain rotationally cold, they get vibrationally hot after each photon absorption, and they undergo a cooling sequence made of successive emission of infrared photons. Due to intrinsic difficulties to mimic such interstellar conditions, no experimental nor theoretical study so far has interrogated the infrared spectroscopy of PAHs under the three above listed characteristics simultaneously fulfilled. 


\subsection{Recent ISO observations}

The recent observations of these infrared emission bands, obtained by pointing ISO to a large variety of astrophysical objects, have revealed the ubiquity of the carriers and the very large stability of the spectral emission pattern. First of all they confirm that most of the infrared intensity is emitted in bands whose wavelengths coincide with those of the fundamental bands of aromatic materials, justifying the new term of AIBs (for Aromatic Infrared Bands). Furthermore they demonstrate that, apart from its absolute integral intensity, the spectral pattern is essentially insensitive to the value of the interstellar radiation field. This beautifully confirms the validity of the single-photon absorption events mechanism, whose consequence is that the carriers must keep a very small size.

Finally a detailed analysis of individual spectra from different types of objects is capable to reveal some interesting variations in the relative intensities of the various bands. These variations are of course the most interesting part for an optimum astrophysical exploitation of the ISO data. However this full exploitation of the results cannot be done without a good understanding of the physical mechanisms responsible for the formation of these emission bands. This requires in particular a detailed knowledge of the spectroscopic properties of the PAHs proposed carriers, in a very wide spectral range (from far IR to far UV), as well as their intramolecular dynamical behavior upon electronic excitation.

\section{The Charge State of PAHs}

The charge state of the interstellar PAHs is an important aspect of this problem. Indeed, the ionization efficiency of $\mathrm{PAHs}$ by interstellar photons is large, and going from neutrals to cationic or anionic PAHs seriously modifies their spectroscopy: infrared intensities are affected, and strong electronic transitions of very small energy appear.

The electronic structure of PAHs is based on the $\mathrm{sp}^{2}$ hybridization of $2 \mathrm{~s}$ and $2 \mathrm{p}$ valence electrons of the carbon atoms, leading to the well known $\pi$-cloud. Removing one electron from this cloud leaves a hole in the bonding orbitals, which produces a large change in the density of electronic states. A distributed charge is present, and the dipole moment becomes then more sensitive to the skeleton vibrations.

It has been realized in recent years that the relative intensities of the various infrared active modes change drastically when the charge state of a PAH is changed. In particular all the C-H bond vibrations tend to dominate the infrared absorption spectra of neutrals, while the $\mathrm{C}-\mathrm{C}$ bond vibrations dominate those of cations. This was shown both experimentally by matrix isolation spectroscopy studies (Allamandola, Hudgins \& Sandford 1999), and theoretically using various approximations in ab-initio calculations (Pauzat, Talbi, \& Ellinger 1995; Bauschlicher \& Langhoff 1997). Very recently we have developed an alternative method, based on the tight-binding (TB) approach to treat the electronic motion by quantum mechanics, and the molecular dynamics (MD) algorithms to run classical trajectories. The calculation of the dipole moment vector along these trajectories, and of its autocorrelation function, leads to the infrared absorption spectrum (Parneix, Nguyen \& Bréchignac 2001). The preliminary results are in good agreement with the previous findings. 


\section{Visible Spectroscopy of Gas Phase PAH Cations}

The laboratory study of these electronic transitions in PAH cations, when achieved in the gas phase, can address questions such as the possible contribution of these species to the Diffuse Interstellar Bands (in the visible and near-IR), and the electronic to vibrational intramolecular energy conversion mechanism, which is an essential feature of the UIB emission process in space.

A new experimental technique capable to produce the electronic absorption spectra of cold gas phase PAH cations has been recently developed in Orsay (Bréchignac \& Pino 1999; Pino, Boudin \& Bréchignac 1999). It consists in measuring the photodissociation spectra of cationic van der Waals complexes, of the form $\mathrm{PAH}^{+}-(\operatorname{argon})_{1,2}$ formed in a molecular beam. Indeed absorption of a near infrared or visible photon by such species results in ejection of the argon atom(s) with a $100 \%$ efficiency. Thus their absorption spectra can be obtained by monitoring the ratio of the fragment ion to parent ion signals at the mass spectrometer detector as a function of the laser wavelength. The binding of an argon atom to a PAH is a small perturbation, which being slightly different in various electronic states can induce a small spectral shift, relative to the transition in the bare molecule/cation. But this shift can be precisely measured by comparing the same transition in $\mathrm{PAH}^{+}-(\operatorname{argon})_{1}$ and $\mathrm{PAH}^{+}-(\operatorname{argon})_{2}$, since these clusters can be chosen so that both argon atoms occupy equivalent binding sites and have the same interaction with the aromatic substrate.

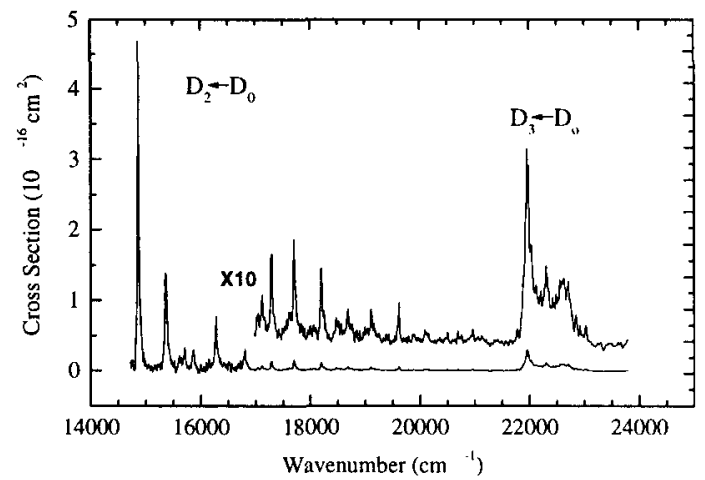

Figure 1. Electronic absorption spectrum of the naphthalene cation.

Among other PAH cations whose near infrared or visible spectra have been recorded thanks to this technique (Bréchignac, Pino \& Boudin 2000 ), the $D_{2}-D_{0}$ transition of Naphthalene ${ }^{+}$(Pino et al. 1999) (see Figure 1) has been used to estimate an upper limit of abundance for this cation along the line-of-sight of HD41117 as $<3 \cdot 10^{-8} \mathrm{~N}_{\mathrm{H}}$ (Boudin, Pino \& Bréchignac 2000).

\section{Intramolecular Dynamics in PAHs}

The bandwidths of the individual vibronic bands in the electronic spectra mentioned above are all larger than usually found for the electronic transitions in the neutral species. These intrinsic bandwidths are the results of lifetime broad- 
ening due to the ultrafast internal conversion processes taking place within the cations, thanks to which the energy, initially injected as electronic energy, is quickly degraded into vibrational energy. This intramolecular energy redistribution is of course the basis for the UV absorption to infrared emission conversion mechanism in PAHs. Even when the whole energy is converted into vibrational energy of the fundamental electronic state, this fast intramolecular dynamics can contribute to the width of the infrared emission bands, as suggested by the most recent high resolution (SWS) ISO observations (Verstraete et al. 2000).

From the new, albeit limited, database on the electronic spectra of cold gas phase PAH cations, it appears that the intramolecular couplings involved in this ultrafast dynamics are affected by the charge state of the molecule: everything else being equal, the square of the coupling matrix element is about 20 times larger in cations relative to neutrals (Pino 1999). This dynamics is also expected to be dependent on the total internal energy content of the molecule, i.e. its microcanonical temperature. Consequently a change in the infrared spectra is also expected: this effect is presently under investigation using the TBMD method, which is particularly well suited for this purpose.

\section{Conclusion}

Very significant progress has been made during the past 15 years in the knowledge and understanding of the spectroscopy of interstellar PAHs. It is clear that the present status is closer than it has never been to the right interstellar conditions. It should hopefully lead us quickly to the point where we could fully exploit the richness of the AIB spectra from the various astrophysical objects.

Acknowledgment : The author wishes to thank the financial support of the Programme National PCMI (CNRS)

\section{References}

Allamadola L.J., Tielens A.G.G.M. and Barker J.R. 1985, ApJ, 290, L25

Allamandola L.J., Hudgins D.M. and Sandford S.A. 1999, ApJ Lett., 511, L115

Bauschlicher C.W. and Langhoff S.R. 1997, Spectrochimica Acta, 53A, 1225

Boudin N., Pino T. and Bréchignac Ph. 2000, J. Mol. structure (in press)

Bréchignac Ph. and Pino T. 1999, A\&A, 343, L49

Bréchignac Ph., Pino T. and Boudin N. 2000, Spectrochimica Acta (in press)

Knacke, R.F. 1977, Nature, 269,132

Léger A. and Puget J.L. 1984, A\&A, 137, L5

Parneix P., Nguyen Thi Van Oanh and Bréchignac Ph. 2001, in preparation

Pauzat F., Talbi D. and Ellinger Y. 1995, A\&A, 293, 263

Pino T., Boudin N. and Bréchignac Ph. 1999, J. Chem. Phys., 111, 7337

Pino T. 1999, PhD thesis, University of Paris-Sud, Orsay (France)

Sellgren K. 1984, ApJ, 277, 623

Verstraete L., Pech C., Moutou C., Sellgren K., Wright C.M., Giard M., Léger A., Timmermann R. and Drapatz S. 2000, A\&A (submitted) 